Western University

Scholarship@Western

Chemistry Publications

Chemistry Department

Winter 3-14-2016

\title{
Poly(ester amide)s with pendant azobenzenes: Multi-responsive self-immolative moieties for modulating polymer assemblies
}

Andrew D. Wong

Western University, awong447@uwo.ca

Alex Prinzen

Western University, alexander.prinzen@mail.mcgill.ca

Elizabeth Gillies

Western University, egillie@uwo.ca

Follow this and additional works at: https://ir.lib.uwo.ca/chempub

Part of the Chemistry Commons

Citation of this paper:

Wong, Andrew D.; Prinzen, Alex; and Gillies, Elizabeth, "Poly(ester amide)s with pendant azobenzenes: Multi-responsive selfimmolative moieties for modulating polymer assemblies" (2016). Chemistry Publications. 88.

https://ir.lib.uwo.ca/chempub/88 


\title{
Journal Name
}

\section{Poly(ester amide)s with Pendant Azobenzenes: Multi-Responsive Self-immolative Moieties for Modulating Polymer Assemblies}

\begin{abstract}
Andrew D. Wong, ${ }^{a}$ Alexander L. Prinzen, ${ }^{b}$ and Elizabeth R. Gillies ${ }^{* a, b}$
Azobenzenes are well-known for their trans-cis photoisomerization, but it was recently demonstrated that azobenzene derivatives could also undergo reduction to trigger a 1,6-elimination and initiate depolymerization of a self-immolative polymer. Herein we explore the optimization of azobenzenes as reduction-sensitive moieties, and their incorporation into functional materials. A library of azobenzenes with electron-withdrawing groups was synthesized, and their rates of reduction by hydrazine were determined. Unexpectedly, a $2-\mathrm{Cl}$ substituent increased the rate of reduction more than other electronegative or sterically-demanding substituents. Next, a new diester monomer containing the 2-Cl-azobenzene was synthesized and incorporated into a poly(ester amide) (PEA) backbone, which was then functionalized with PEO to afford an amphiphilic multi-responsive material. The photo- and reduction-sensitivity of the azobenzenes was then exploited to produce reversible and irreversible changes to the polymer nanoassemblies in water. Their responsiveness to light and/or hydrazine was studied by ultraviolet-visible (UV-Vis) spectroscopy, dynamic light scattering (DLS), and fluorescence spectroscopy of encapsulated nile red. Alternating irradiation with UV and visible light resulted in reversible trans-cis isomerization, which changed the polarity of the micelle core without disrupting the assemblies. Reduction by hydrazine resulted in the release of nile red from the micelle core, while residual assemblies were still detected by DLS, likely due to the presence of remaining hydrophobes. A combination of UV light and hydrazine resulted in the release of nile red and breakdown of the assemblies. These results suggest that the intrinsic responsiveness of azobenzene to both light and reductive stimuli can provide polymer assemblies that respond to one or more stimuli in unique and synergistic ways through a single multi-responsive unit.
\end{abstract}

\section{Introduction}

Stimuli-responsive materials possess properties that change upon exposure to one or more stimuli. ${ }^{1-3}$ Stimuli such as light, ${ }^{4}$ ${ }^{5}$ or changes in redox potential, ${ }^{6,7} \mathrm{pH}^{8-10}$ temperature, ${ }^{11,12}$ or magnetic fields ${ }^{13}$ have been successfully employed to induce reversible or irreversible changes in the chemical, biological, mechanical, or electrical properties of materials. These changes can be exploited to provide functions in a wide range of applications. For example, stimuli-responsive drug carriers can release their payloads in response to the stimulus, affording increased selectivity for their target. ${ }^{14,}{ }^{15}$ Responsiveness to multiple stimuli, both separately or simultaneously, can extend the versatility of stimuli-responsive materials, and increase their sensitivity. ${ }^{16,17}$

Azobenzene derivatives have been widely used as photoswitches both in the context of small molecules and polymers due to their highly efficient and reversible trans-cis

\footnotetext{
${ }^{a .}$ Department of Chemical and Biochemical Engineering, The University of Western Ontario, 1151 Richmond St., London, Canada N6A 5B9

${ }^{b}$. Department of Chemistry, The University of Western Ontario, 1151 Richmond St., London, Canada N6A 5B9. E-mail: egillie@uwo.ca.

Electronic Supplementary Information (ESI) available: additional experimental

procedures, ${ }^{1} \mathrm{H}$ NMR spectra, ${ }^{13} \mathrm{C}$ NMR spectra, SEC traces, additional DLS data. See DOI: $10.1039 / x 0 \times x 00000 x$
}

isomerization upon exposure to UV or visible light. ${ }^{18-20}$ Isomerization of the diazene bond from the trans to cis conformation results in a significant increase in its polarity, ${ }^{21}$ which has been exploited in the context of stimuli-responsive materials. ${ }^{4,22}$ For example, polymer micelles and vesicles prepared from block copolymers containing azobenzene groups in their hydrophobic blocks were introduced, using hydrophilic blocks such as poly(acrylic acid), ${ }^{23}, 24$ poly(ethylene oxide $)^{25}$ or even in linear-dendritic systems. ${ }^{26}$ Isomerization of the azobenzene moieties from trans to cis in response to irradiation with UV light destabilized the assemblies. Upon irradiation with visible light, the amphiphilic nature of the polymer was restored, and the assemblies were re-formed in situ. These assemblies underwent organizational changes in response to light, but did not degrade as they were composed of stable polymer backbones.

In recent work, we have demonstrated that in addition to their ability to undergo isomerization, appropriately designed azobenzene molecules could also be used as reductionsensitive end-caps/triggers to initiate the end-to-end depolymerization of self-immolative polymers (SIPs). ${ }^{27}$ It was shown that reduction of the azobenzene to hydrazobenzene generated a species capable of a 1,6-elimination reaction, triggering the depolymerisation of a linear self-immolative polycarbamate. The reduction was accompanied by the 


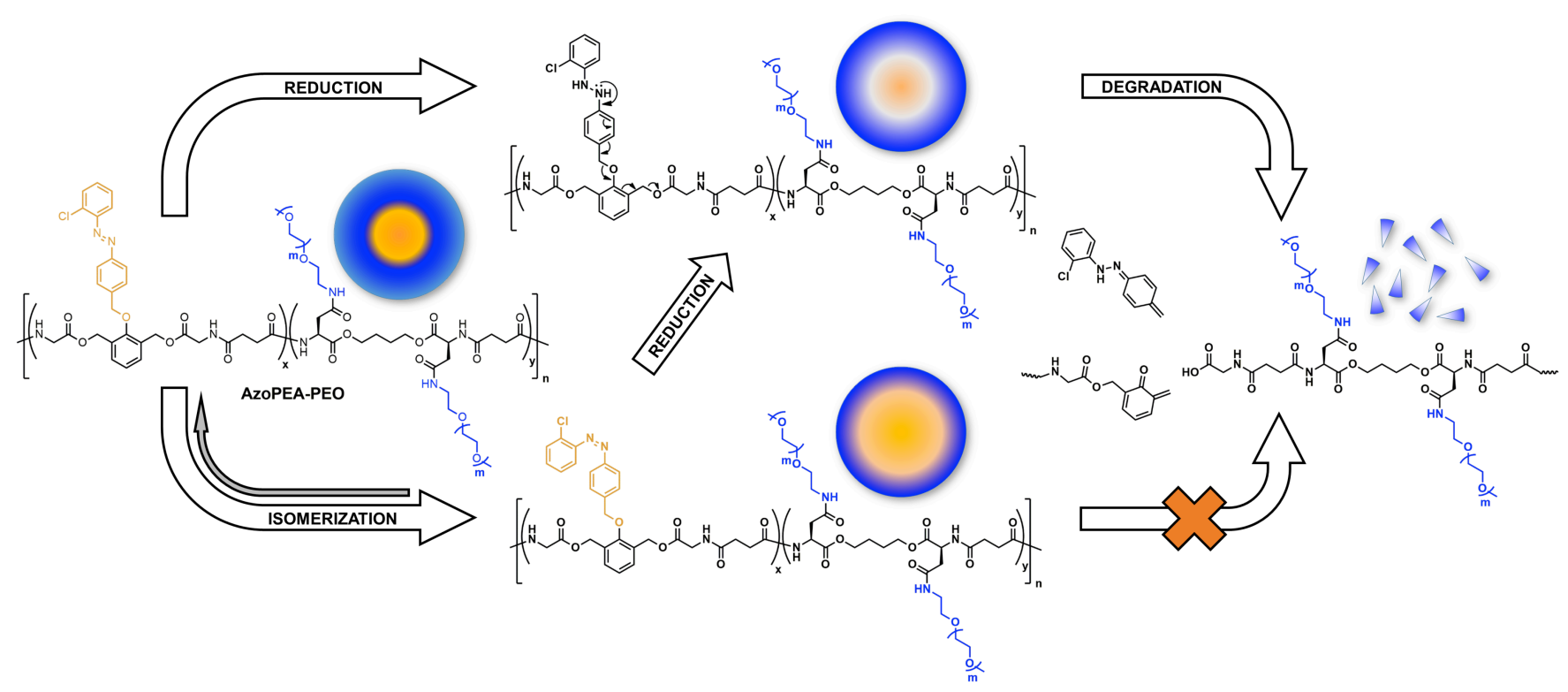

Fig. 1. Schematic demonstrating the proposed multi-stimuli responsiveness of the azobenzene-PEA-PEO system. Reduction of the polymer assemblies leads to irreversible polymer degradation via a cascade of self-immolative elimination reactions, while isomerization with UV light leads to a reversible change in the polarity of the core of the assemblies.

disappearance of the characteristic strong absorbance of the azobenzene, providing a colorless solution and thus a visual cue that the end-cap had been activated. However, this system could not be investigated in water due to the hydrophobicity of the polymer backbone. Furthermore, the potential effects of light-mediated trans-cis isomerization on the system were not explored.

Poly(ester amide)s (PEAs) are polymers that contain both ester and amide functional groups within their backbones. ${ }^{28,29}$ PEAs prepared from diols, diacids, and amino acids are a particularly attractive class of PEAs as the combination of monomers can be easily tuned to afford a wide range of properties and functions. ${ }^{30-40}$ They are of interest for biomedical applications ranging from drug delivery to tissue engineering because they are degradable under physiological conditions, and have been found to be non-toxic and to support cell growth. ${ }^{41-49}$ Previous research in our group has demonstrated that it is possible to prepare PEA-poly(ethylene oxide) (PEO) graft copolymers through derivatization of the pendant groups of amino acids such as aspartic acid or lysine along the PEA backbone. ${ }^{50}$ The resulting amphiphilic copolymers could be assembled into micelles that released drugs such as paclitaxel in a sustained and/or stimuliresponsive manner. ${ }^{51,52}$

The modularity and versatility of the PEA structure also affords the opportunity to incorporate azobenzene moieties in a manner that can allow their responsiveness to light and reduction to be exploited both separately and synergistically. Described here is first the optimization of the azobenzene chemical structure to afford increased reduction rates. Subsequently, the optimized azobenzene is incorporated as a pendant group on a PEA-PEO graft copolymer and this copolymer is self-assembled to form nanoparticles in aqueous solution. As shown in Fig. 1, reduction of the azobenzenes is designed to lead to backbone fragmentation via a selfimmolative 1,6- then 1,4-elimination reaction. On the other hand, trans-cis isomerization is designed to change the polarity of the assembly core. Synergistically, isomerization can potentially increase the rate of breakdown of the polymer by increasing the polarity of the assembly core, providing enhanced penetration by the reducing agent and water. These changes are probed using UV-visible (UV-vis) spectroscopy, dynamic light scattering (DLS), and the fluorescence of nile red as a probe molecule. To the best of our knowledge, this is the first example exploiting both the photo- and reductionresponsiveness of azobenzene. It is shown that the unique capabilities of azobenzene enable access to both reversible and irreversible disruption of polymer assemblies.

\section{Results and Discussion}

\section{Optimization of the azobenzene reduction rate}

In our previous study, an electron-withdrawing ester group was incorporated onto the azobenzene with the aim of promoting reduction. ${ }^{27}$ However, the azobenzene structure was not optimized with respect to the rate of reduction. As depolymerisation of the self-immolative polycarbamate requires days, ${ }^{27,53,54}$ end-cap reduction was not rate-limiting in the process and the specific azobenzene structure did not play a significant role in the overall rate of polymer degradation. On the other hand, in the current design (Fig. 1), many azobenzene reductions are required to completely degrade the polymer, so there was motivation for investigating and optimizing the rate of reduction by tuning the chemical structure of the azobenzene. To achieve this, azobenzenes 1 through 12 (Fig. 2) were synthesized by the general route shown in Scheme 1, involving oxidation of the appropriate 


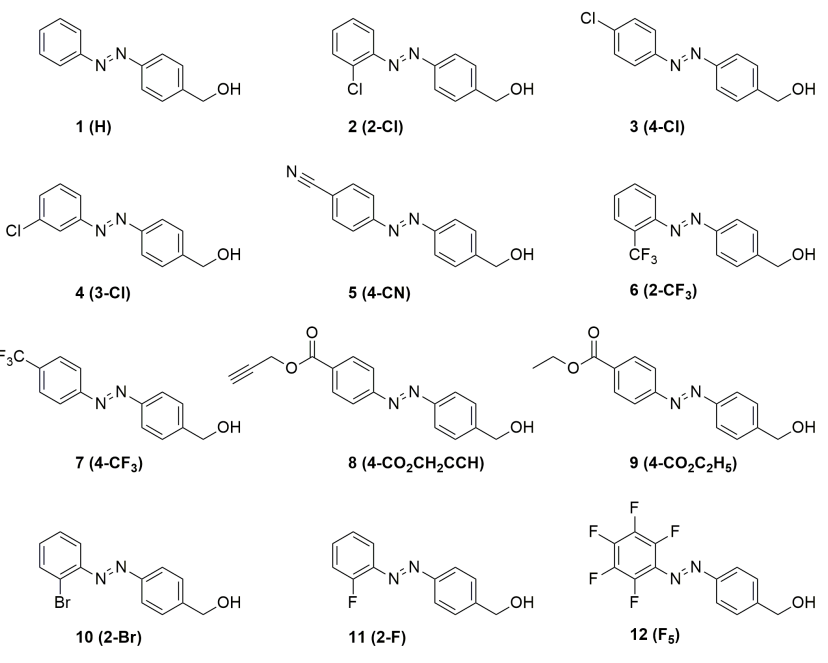

Fig. 2. Library of reduction-sensitive azobenzene derivatives synthesized in this study.

starting aniline to its corresponding nitrosobenzene, followed by condensation with 4-aminobenzyl alcohol in the presence of acetic acid $(\mathrm{AcOH})$. Nitrosobenzene intermediates were not isolated due to their instability, and were used for the subsequent step immediately following aqueous workup. All of the derivatives except $\mathbf{1}$ were designed to contain electronwithdrawing groups, as this has previously been shown to enhance the rate of reduction. ${ }^{55}$

To study the reduction kinetics, a $25 \mu \mathrm{M}$ solution of each azobenzene in $3 / 5$ tetrahydrofuran (THF)/water mixture was prepared and $250 \mu \mathrm{M}$ hydrazine hydrate was added as a reducing agent (

Scheme 1). The water/THF mixture was used in order to study the reduction in a primarily aqueous environment, while maintaining solubility of the hydrophobic azobenzene molecules using THF. The 10-fold excess of hydrazine was used to provide pseudo-first-order conditions to facilitate the kinetic analysis. The reduction in absorption (A) of each azobenzene derivative at its maximum absorption wavelength $\left(\lambda_{\max }\right)$ corresponding to the $\pi-\pi^{*}$ transition ( $323-336 \mathrm{~nm}$ ) was monitored over 10 minutes using UV-vis spectroscopy. This technique was chosen due to the hypsochromic shift in absorbance between the decreasing azobenzene peak and growing hydrazobenzene peak (Fig. 3). Plots of $\ln \left(A / A_{0}\right)$ versus time (ESI, Fig. S1) were used to determine the observed rate constant of reduction $\left(k_{\text {obs }}\right)$ for each derivative and the results are shown in Table 1.

The different azobenzene derivatives exhibited $k_{\text {obs }}$ for reduction ranging from $1.4 \times 10^{-2} \mathrm{~min}^{-1}$ to $8.0 \times 10^{-2} \mathrm{~min}^{-1}$, demonstrating that the rate could be tuned over almost one order of magnitude, depending on the substitution on the aromatic rings. Nearly all derivatives were reduced more rapidly than the unfunctionalized azobenzene 1 . The introduction of a halogen at the 2-position on the aromatic ring was most effective in increasing the reduction rate. Compound $2(2-\mathrm{Cl})$ was the most reduction-sensitive azobenzene, exhibiting a nearly four-fold increase in rate over the unfunctionalized azobenzene 1 and about a two-fold

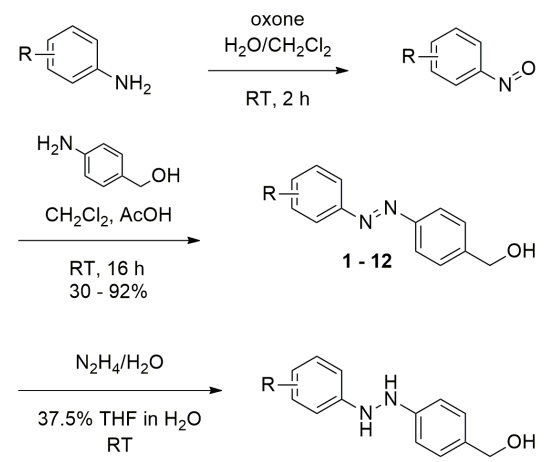

Scheme 1. General two-step synthesis of azobenzene derivatives $\mathbf{1}$ - 12, and their reduction by hydrazine hydrate in THF/water (3/5).

Table 1. $\mathrm{k}_{\mathrm{obs}}$ for the reduction of azobenzene derivatives 1 - 12

\begin{tabular}{lc}
\hline Compound & $\mathbf{k}_{\text {obs }}\left(\mathbf{x} 10^{-2} \mathbf{m i n}^{-1}\right)$ \\
\hline $\mathbf{1}(\mathrm{H})$ & $2.2 \pm 0.4$ \\
$\mathbf{2}(2-\mathrm{Cl})$ & $8.0 \pm 1.1$ \\
$\mathbf{3}(4-\mathrm{Cl})$ & $2.5 \pm 0.4$ \\
$\mathbf{4}(3-\mathrm{Cl})$ & $2.3 \pm 0.2$ \\
$\mathbf{5}(4-\mathrm{CN})$ & $2.9 \pm 0.2$ \\
$\mathbf{6}\left(2-\mathrm{CF}_{3}\right)$ & $2.6 \pm 0.2$ \\
$\mathbf{7}\left(4-\mathrm{CF}_{3}\right)$ & $3.2 \pm 0.4$ \\
$\mathbf{8}\left(4-\mathrm{CO}_{2} \mathrm{CH}_{2} \mathrm{CCH}\right)$ & $2.5 \pm 0.2$ \\
$\mathbf{9}\left(4-\mathrm{CO}_{2} \mathrm{CH}_{2} \mathrm{CH}_{3}\right)$ & $4.4 \pm 0.3$ \\
$\mathbf{1 0}(2-\mathrm{Br})$ & $6.0 \pm 0.8$ \\
$\mathbf{1 1}(2-\mathrm{F})$ & $4.8 \pm 0.4$ \\
$\mathbf{1 2}\left(\mathrm{F}_{5}\right)$ & $1.4 \pm 0.1$ \\
\hline
\end{tabular}

increase over that of our previously studied compound 9 (4$\left.\mathrm{CO}_{2} \mathrm{CH}_{2} \mathrm{CH}_{3}\right)$. Compound $10(2-\mathrm{Br})$ was more rapidly reduced than $11(2-\mathrm{F})$, but more slowly than $2(2-\mathrm{Cl})$. The difference in reduction rates between these three compounds was analyzed in terms of electronic and steric factors. Fluorine is the most electronegative $(\mathrm{EN})$ atom $\left(\mathrm{EN}_{\mathrm{F}}=4.0, \mathrm{EN}_{\mathrm{Cl}}=3.0, \mathrm{EN}_{\mathrm{Br}}=2.8\right)$, so the inductive withdrawing effect was expected to be much larger than for the other halogens. However, the orbital overlap of fluorine with the aromatic $\pi$ system is high due to its $2 p$ orbitals, resulting in stronger $\pi$-donation than for the larger halogens. ${ }^{56}$

While Hammett parameters $(\sigma)$ do not exist for ortho substituents due to complicating effects associated with steric hindrance, the competing $\sigma$-withdrawing and $\pi$-donation effects of the halogens are reflected in the $\sigma$ values of halogens as para-substituents $\left(\sigma_{\mathrm{F}}=0.06, \sigma_{\mathrm{Cl}}=0.23, \sigma_{\mathrm{Br}}=\right.$ $0.23)^{57}$ In the current reaction, ring torsion would be expected to accelerate the reduction rate by raising the energy of the starting material relative to that of a completely conjugated 
azobenzene. Bromine has the largest radius $(r)$ of the evaluated halogens $\left(r_{F}=71 \mathrm{pm}, r_{\mathrm{Cl}}=99 \mathrm{pm}, \mathrm{r}_{\mathrm{Br}}=114 \mathrm{pm}\right)$ and would be expected to have the highest impact on ring torsion. However, $10(2-\mathrm{Br})$ was reduced more slowly than $\mathbf{2}(2-\mathrm{Cl})$. This indicates that ring torsion does not play a dominant role in accelerating the reduction rate for this series of azobenzenes.

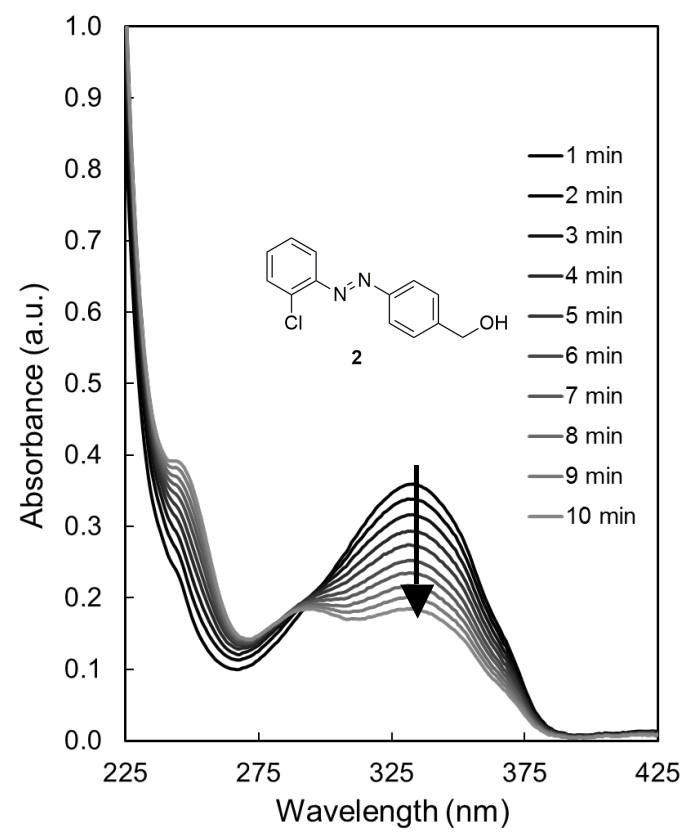

Fig. 3. The observed decrease in absorbance of compound $\mathbf{2}$ upon reduction by hydrazine, as measured by UV-Vis spectroscopy ( $25 \mu \mathrm{M} 2$ and $250 \mu \mathrm{M}$ hydrazine in THF/water 3/5).

The role of electronics and sterics was further explored by comparing the reduction rates of $\mathbf{6}\left(2-\mathrm{CF}_{3}\right)$ and the $\mathbf{2}(2-\mathrm{Cl})$. The Hammett parameter for the trifluoromethyl group is significantly larger than for chlorine $\left(\sigma_{\mathrm{Cl}}=0.23, \sigma_{\mathrm{CF} 3}=0.54\right)$ as there can be no $\pi$-donation to the ring. Furthermore, the $\mathrm{CF}_{3}$ group is much larger than $\mathrm{Cl}^{58}$ and was thus expected to cause greater ring torsion. Despite all of these factors that should favour more rapid reduction of $6\left(2-\mathrm{CF}_{3}\right)$, it was reduced more slowly than $2(2-\mathrm{Cl}) .7\left(4-\mathrm{CF}_{3}\right)$ was reduced at a similar rate to 6 $\left(2-\mathrm{CF}_{3}\right)$. However, $3(4-\mathrm{Cl})$ and $4(3-\mathrm{Cl})$ were reduced more slowly than $2(2-\mathrm{Cl})$, demonstrating that the ortho position is critical for the $\mathrm{Cl}$ substituent.

The introduction of various other electron-withdrawing substituents such as esters (8 and 9) or a cyano (4) at the 4position also afforded intermediate rates of reduction and there was no significant correlation in the reduction rates of the 4-derivatives with the Hammett values for these substituents (Fig. S2). Surprisingly the perfluorinated derivative 12 was reduced more slowly than all other derivatives and even the unfunctionalized azobenzene 1, despite its presumed electron deficiency.

Considering the data as a whole, there appears to be a unique feature of $\mathbf{2}(2-\mathrm{Cl})$ that results in its more rapid reduction relative to other derivatives with more electrondeficient character and/or greater torsional strain. We propose that the results may be explained by halogen bonding. Recent studies $^{59}$ have shown that aryl halogens are highly anisotropic in their electron distribution, leading to a $\delta+$ region $\sim 180^{\circ}$ from the $\sigma$-bond. The presence of a halogen $(X)$ at the 2-position may favor the association of hydrazine with the azobenzene via an $\mathrm{N}(\delta-)-\mathrm{X}(\delta+)$ dipole-dipole interaction, effectively preorganizing the reagent complex for reduction (Fig. S3). This behaviour is not often observed with aryl fluorides, and the effect is stronger for $\mathrm{Cl}$ than for $\mathrm{Br}$, explaining the observed trend in reduction rates. ${ }^{59}$

\section{Synthesis of an azobenzene-containing PEA}

To demonstrate the potential of azobenzene as a multi-stimuli responsive moiety, the most rapidly-reduced azobenzene 2 was incorporated as a pendant group on an amphiphilic PEAPEO graft copolymer. The synthesis of a diester monomer incorporating an azobenzene was accomplished according to Scheme 2. First, azobenzene derivative $\mathbf{2}$ was reacted with carbon tetrabromide and triphenylphosphine in an Appel reaction to afford the benzylic bromide 13. A phenoxide was then prepared from $14,{ }^{60}$ and was subsequently alkylated with 13, affording the dialdehyde $\mathbf{1 5}$. The aldehydes of $\mathbf{1 5}$ were then selectively reduced in the presence of the diazene bond to benzylic alcohols using $\mathrm{NaBH}_{4}$ to give compound 16. Two equivalents of $\mathrm{N}$ - $t$-butyloxycarbonyl (Boc)-protected glycine were then coupled to $\mathbf{1 6}$ using carbonyl diimidazole (CDI) to afford 17. The Boc group was removed from compound $\mathbf{1 7}$ using trifluoroacetic acid (TFA) directly before polymerization to yield the target monomer 18.

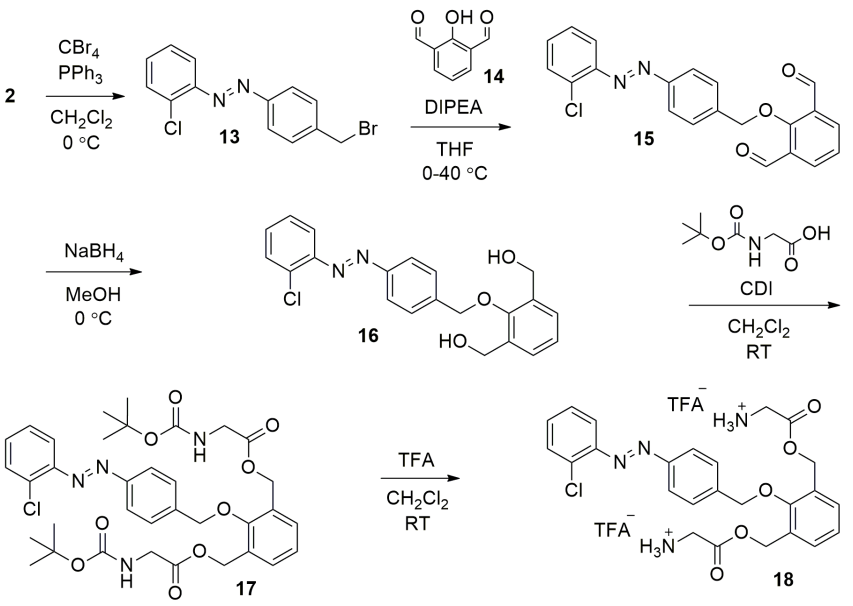

Scheme 2. Synthesis of an azobenzene-containing diester monomer.

As shown in Scheme 3, monomers $18,19,^{36}$ and $20^{37}$ (0.9/0.1/1.0), were then reacted via a condensation polymerization in $\mathrm{N}, \mathrm{N}$-dimethylacetamide (DMA) to give polymer $\mathbf{P 1}$. This polymer had a weight-average molar mass $\left(\mathrm{M}_{\mathrm{w}}\right)$ of $7200 \mathrm{~g} \mathrm{~mol}^{-1}$ and a dispersity $(\Theta)$ of 2.26 as measured by size exclusion chromatography (SEC) relative to poly (methyl methacrylate) standards. The 0.1 equiv. of the aspartic acidbased monomer 19 were incorporated to provide attachment sites for PEO chains following cleavage of the $t$-butyl ester protecting groups. Thus, the pendant carboxylic acid groups of P1 were deprotected using TFA and subsequently coupled to 
amine-terminated poly(ethylene oxide) monomethyl ether (PEO-NH $\mathrm{NH}_{2} 2000 \mathrm{~g} \mathrm{~mol}^{-1}$ ) yielding the target amphiphilic graft copolymer, P2. This polymer had an $\mathrm{M}_{\mathrm{w}}$ of $5900 \mathrm{~g} \mathrm{~mol}^{-1}$ and a $\nexists$ of 2.13 as measured by SEC relative to PMMA. It was surprising that $\mathbf{P 2}$ had a lower molar mass than its precursor P1 but this phenomenon has been previously observed by SEC for PEA-PEO graft copolymers and may be related to conformational effects. ${ }^{39,61}$ Using ${ }^{1} \mathrm{H}$ NMR spectroscopy, based on the relative integrations of the PEO peak at $3.5 \mathrm{ppm}$ and the peak at $3.9 \mathrm{ppm}$ corresponding to the $\alpha$-hydrogens of glycine on the azobenzene monomer, the graft copolymer that was 42 wt\% PEO. A control polymer P3 (Fig. 4 and ESI Scheme S1) without azobenzene moieties was also prepared in order to demonstrate that any changes in properties arise from triggering of the azobenzene moieties rather than non-specific cleavage of the esters in the polymer backbone. This polymer had a $\mathrm{M}_{\mathrm{w}}$ of $18200 \mathrm{~g} \mathrm{~mol}^{-1}, \ominus$ of 1.58 , and a PEO content of 34 wt\%.
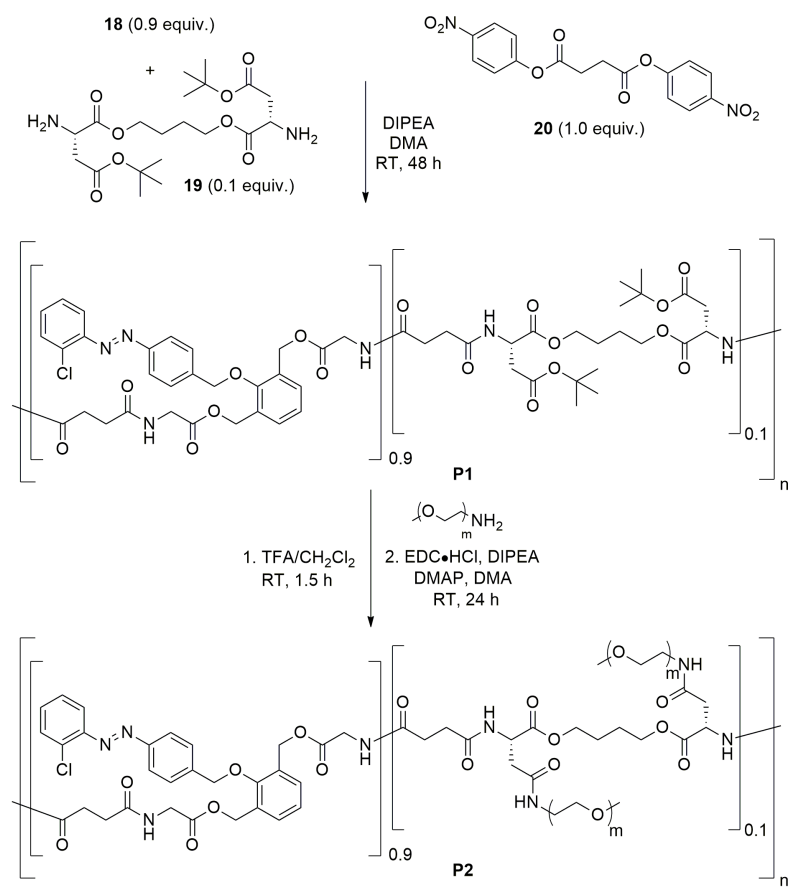

Scheme 3. Synthesis of a PEA having pendant azobenzenes, and post-polymerization conjugation of PEO to afford an amphiphilic graft copolymer.

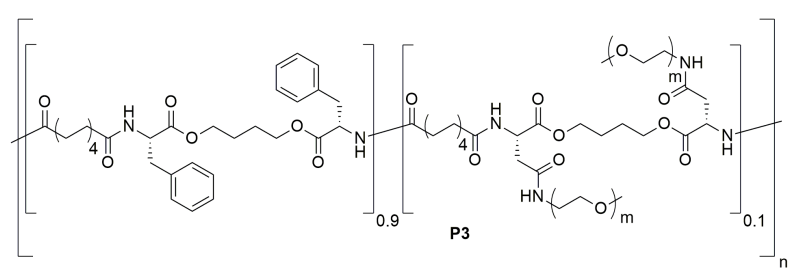

Fig. 4. Control polymer P3 synthesized for comparison with P2.

\section{Self-assembly of P2 and P3}

Nanoassemblies were prepared from the stimuli-responsive polymer $\mathbf{P 2}$ and from the control polymer $\mathbf{P} \mathbf{3}$ by the rapid addition of a solution of polymer in DMSO to water. The suspensions were then diluted four-fold with water and dialysed against a $1 \mathrm{~kg} \mathrm{~mol}^{-1}$ molecular weight cut-off (MWCO) membrane in water to remove the organic solvent. The Zaverage hydrodynamic diameters of the resulting assemblies were determined by DLS to be $\sim 90 \mathrm{~nm}$ for P2 (Fig. 5A) and $\sim 100 \mathrm{~nm}$ for P3 (ESI, Fig. S39A). The assemblies were also imaged by transmission electron microscopy (TEM). As shown in Fig. 5B and Fig. S39B, the assemblies were solid particles, suggesting that they were micelles or compound micelles.
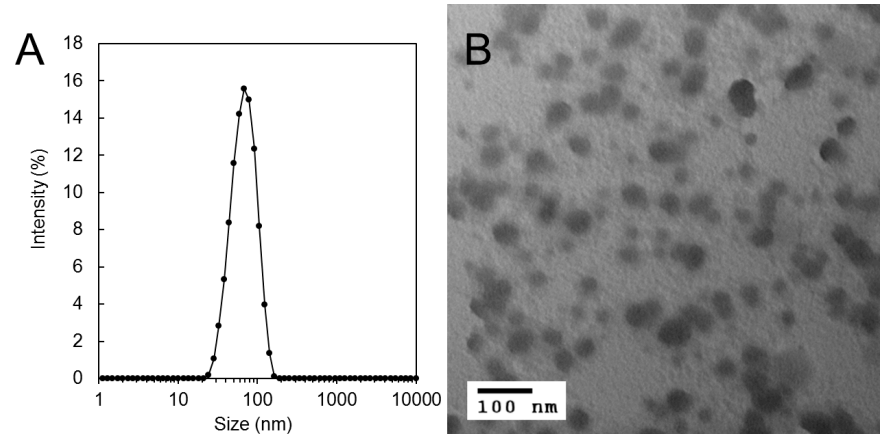

Fig. 5. A) DLS trace showing the intensity distribution of $\mathbf{P} \mathbf{2}$ nanoassemblies in aqueous solution. B) TEM images of $\mathbf{P} \mathbf{2}$ nanoassemblies.

\section{Stimuli-responsive properties of azobenzene-PEA assemblies}

First, the UV-responsive properties of the micelles were probed. Irradiation with light was expected to result in reversible changes in the micellar structure. Alternating irradiation with UV and visible light was used to convert the pendant azobenzenes between the trans and cis conformations and the effects were measured by fluorescence and UV-Vis spectroscopy. As shown in Fig. 6, $10 \mathrm{~min}$ of irradiation with UV light resulted in a $\sim 35 \%$ decrease in the absorbance at $330 \mathrm{~nm}$ corresponding to the trans conformation. Irradiation for $10 \mathrm{~min}$ with visible light resulted in an increase in the absorbance at $330 \mathrm{~nm}$ back to its original level. There were no changes in the maximum and minimum absorbance over 8 cycles, confirming that the isomerization was fully reversible.

To further probe the effects of the isomerization on the micelles, 5 wt\% of nile red as a probe molecule was encapsulated in the micelles by dissolving nile red in the DMSO polymer solution, and then forming the assemblies as described above. The same cycles of irradiation with UV and visible light were repeated. As shown in Fig. 6, irradiation with UV light for $10 \mathrm{~min}$ resulted in a $\sim 15 \%$ reduction in the nile red fluorescence. It is well established that the fluorescence intensity of nile red is strongly dependent on its environment, with increased fluorescence observed in more hydrophobic environments and reduced fluorescence in more polar environments due aggregation and consequent quenching. ${ }^{63,64}$ Therefore, the reduction in nile red fluorescence upon transcis isomerization likely results from an increase in polarity of the micelle core, increased water penetration into the micelle core resulting from this polarity, some release of nile red from the micelle into the bulk water or some combination of these phenomena. This process was also reversible upon irradiation with visible light, resulting in an increase in nile red 
fluorescence back to approximately its initial value. It was noted that the fluorescence varied to some extent with each cycle, suggesting that some minor reorganization of the micelles and the encapsulated nile red might have occurred as a result of the isomerization. Nevertheless, the process was clearly reversible.

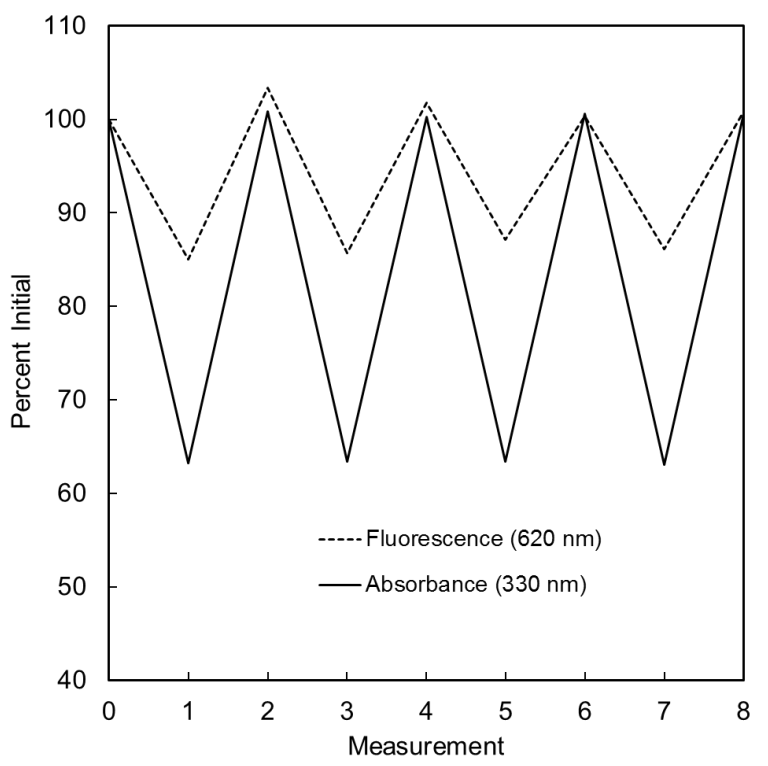

Fig. 6. Reversible increase and decrease in absorbance of the trans-azobenzene and fluorescence of encapsulated nile red in $\mathbf{P 2}$ assemblies upon irradiation with UV and visible light in alternating 10 min cycles.

DLS was also used to the study the effect of light irradiation on the assemblies. In this experiment, the assemblies were irradiated continuously with UV light. As shown in Fig. 7, after $8 \mathrm{~h}$ of irradiation, a $\sim 25 \%$ reduction in the mean count rate was observed. The count rate in light scattering is proportional to the number, size, and density of the aggregates. No obvious changes in the particle size distribution were observed (Fig. S45). Therefore, in agreement with the nile red fluorescence results, this suggests that the particles just underwent some subtle reorganization. Micelles of $\mathbf{P 2}$ without any stimulus did not exhibit any significant changes in the mean count rate.

Unlike trans-cis isomerization, treatment with hydrazine was expected to result in irreversible disruption of the micelles upon bond cleavage initiated by azobenzene reduction followed by 1,6- and 1,4-elimination reactions. Micelles of P2 containing nile red were treated with $250 \mu \mathrm{M}$ hydrazine. As shown in Fig. 8, this resulted in a $\sim 80 \%$ decrease in nile red fluorescence over a period of 9 hours. This large decrease in nile red fluorescence is consistent with breakdown of the micelles and release of the nile red into the aqueous environment. In the absence of hydrazine, $<5 \%$ decrease in fluorescence was observed. Control micelles prepared from P3 were also treated with $250 \mu \mathrm{M}$ hydrazine. This resulted in a $\sim 30 \%$ reduction in nile red fluorescence, which can likely be

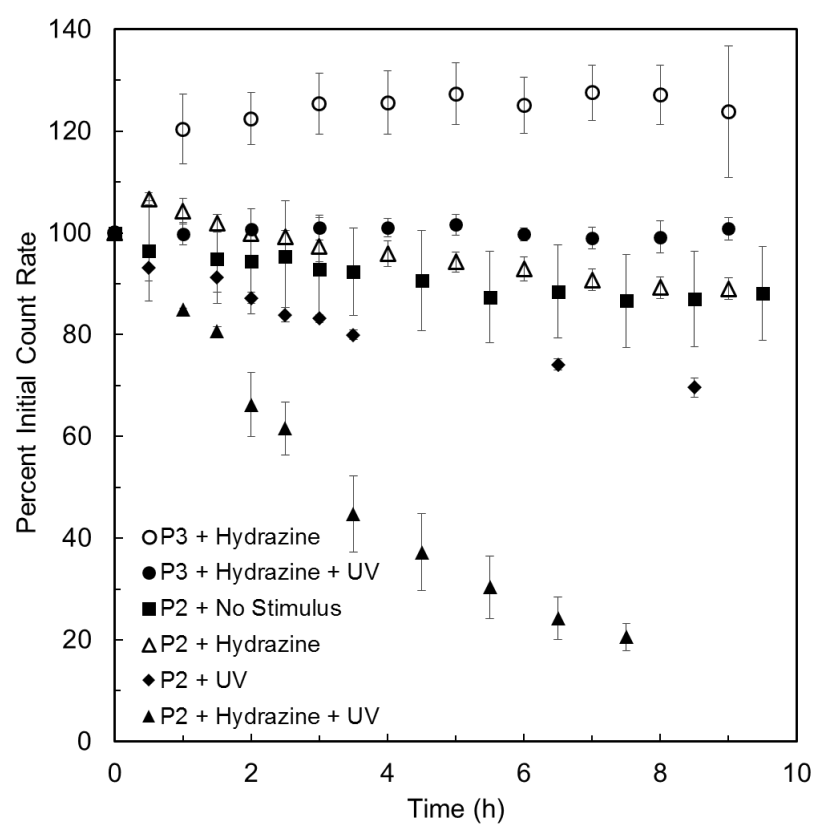

Fig. 7. Changes in the mean count rate measured by DLS for assemblies of $\mathbf{P} \mathbf{2}$ and $\mathbf{P} \mathbf{3}$ under various conditions.

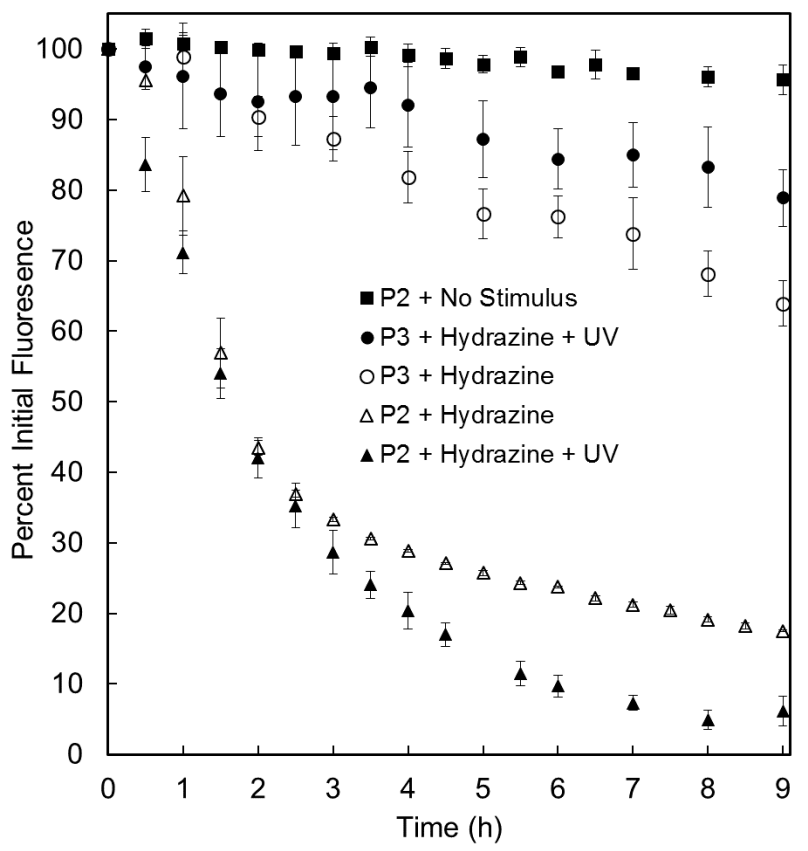

Fig. 8. Changes in the observed fluorescence of nile red-containing polymer assemblies of P2 and P3.

attributed to a small amount of ester cleavage induced by hydrazine, which is strongly nucleophilic. However, this degradation was much less than that of $\mathbf{P 2}$, confirming that the observed effects for $\mathbf{P 2}$ primarily resulted from bond cleavage induced by azobenzene reduction.

The effects of hydrazine on the DLS count rate were also investigated. As shown in Fig. 7, assemblies of $\mathbf{P 2}$ exhibited an initial small increase in the mean count rate immediately 
following hydrazine addition and this was followed by a small gradual decrease. The initial increase can likely be attributed to scattering by hydrazine. Although it is a small molecule that does not strongly scatter light, its concentration is $\sim 10$-fold higher than that of the polymer in terms of molarity. It was somewhat surprising that the mean count rate did not decrease more substantially over time, given the large changes in nile red fluorescence observed upon reduction. Examination of the assembly size distributions at different time points during the experiment (Figure S43) did not reveal any significant changes. This suggests that the degradation products have some susceptibility to aggregation due to the presence of residual hydrophobes following backbone cleavage events, though these assemblies were clearly less effective at encapsulating nile red and/or are much less hydrophobic. The addition of $250 \mu \mathrm{M}$ hydrazine to assemblies of $\mathbf{P 3}$ led to an initial $\sim 30 \%$ increase in the mean count rate, likely attributable to scattering by hydrazine, and this count rate remained relatively stable over 9 hours (Fig. 7), again demonstrating the specific effect of hydrazine on the azobenzene-containing assemblies.

Finally, the combination of light and hydrazine as stimuli was investigated. As shown in Fig. 8, when the $\mathbf{P 2}$ assemblies were treated with hydrazine and irradiated with UV light for 10 minutes, a $>90 \%$ decrease in fluorescence in of nile red was observed over 9 hours. This decrease was larger than that observed for hydrazine alone, suggesting that the trans-cis isomerization provides a synergistic effect. Isomerization to the cis azobenzene may increase the polarity of the micelle core, enabling better penetration by hydrazine and/or water. Control P3 assemblies were also subjected to the same hydrazine and UV treatment and the decrease in nile red fluorescence was only $\sim 20 \%$. By DLS, more than $\sim 80 \%$ decrease in the mean count rate was observed for P3 assemblies over less than 8 hours, a much larger decrease than was observed for hydrazine alone. This result suggests that trans-cis isomerization may somehow assist in the disruption of the aggregates remaining from hydrazine treatment alone, providing a synergistic effect on the DLS results. In contrast, treatment of control P3 micelles with both UV light and hydrazine did not lead to any significant change in the DLS count rate. This result again confirms the specific role of azobenzene in both light- and hydrazine-mediated disruption of the micelles.

\section{Conclusions}

The rate of azobenzene reduction was optimized through the preparation and study of a library of twelve electron-deficient azobenzenes. It was found that the reduction-sensitivity of these compounds was tunable, with a 2-chloro derivative affording the fastest reduction rate of the studied compounds. This result was attributed to a halogen bonding effect that may promote precomplexation of the azobenzene with the reducing agent. 2-Chloroazobenzenes were then successfully incorporated as pendant groups on an amphiphilic PEA-PEO graft copolymer. This copolymer was assembled into micellar- like structures in aqueous solution. A combination of UV-vis spectroscopy, fluorescence spectroscopy using nile red as a probe, and DLS were used to probe the responses of the micelles to trans-cis isomerization triggered by light, reduction triggered by hydrazine, and a combination of light and reductive stimuli. It was found that light imparted reversible changes in the micelle core, whereas hydrazine led to an irreversible reduction in nile red fluorescence. A combination of light and reductive stimuli led to more significant changes in nile red fluorescence and in mean DLS count rate than either stimulus alone, suggesting a synergistic effect. In contrast, control samples of PEA micelles without azobenzene and azobenzene micelles without stimuli only underwent small changes in nile red fluorescence and DLS count rate either in the presence or absence of stimuli. Thus, this work provides the first example demonstrating the response of azobenzene to multiple stimuli, both separately and synergistically and demonstrates that this response can be translated into both reversible and irreversible changes in the properties of materials.

\section{Experimental}

\section{General Procedure and Materials}

Chemicals were obtained from Alfa Aesar, Sigma Aldrich, and AK Scientific, and were used without further purification unless otherwise noted. Solvents were of reagent grade and obtained from Caledon and Fisher. They were used without further purification unless otherwise noted. Spectroscopy-grade THF was obtained from Fisher Scientific. DMA was refluxed over calcium hydride for 12 hours and freshly distilled prior to use. $\mathrm{CH}_{2} \mathrm{Cl}_{2}$ was freshly distilled over $\mathrm{CaH}_{2}$. DMSO for nanoassembly preparation was distilled in glass grade and was obtained from Caledon. Deionized (DI) water was obtained using a Barnstead EASYpure $^{\circledR}$ II system. Dialysis was carried out using regenerated cellulose membranes (Spectra/Por ${ }^{\circledR} \mathrm{RC}$ ) with molecular weight cutoffs (MWCO) of $1 \mathrm{~kg} \mathrm{~mol}^{-1}$ or $50 \mathrm{~kg} \mathrm{~mol}^{-1}$. Compounds $1,^{65} \mathbf{9},{ }^{27} \mathbf{1 4},{ }^{60} \mathbf{1 9},{ }^{36}$ and $\mathbf{2 0}^{37}$ were prepared as previously reported. Thin layer chromatography (TLC) was run on Macherney-Nagel Polygram SIL G/UV254 plates and SiliaFlash P60 silica (40-60 $\mu \mathrm{m}, 230-400$ mesh) was used for column chromatography. ${ }^{1} \mathrm{H}$ NMR and ${ }^{13} \mathrm{C}$ NMR spectra were obtained using 600 or $400 \mathrm{MHz}$ Varian Inova spectrometers. NMR chemical shifts are reported in ppm and are calibrated against residual solvent signals $(\delta \mathrm{H}, \mathrm{C})$ of $\mathrm{CDCl}_{3}(\delta 7.26 \mathrm{ppm}$, $77.2 \mathrm{ppm}$ ), $\mathrm{CD}_{3} \mathrm{OD}$ ( $\delta 3.31 \mathrm{ppm}, 49.2 \mathrm{ppm}$ ) or DMSO- $d_{6}(\delta 2.50$ ppm, $39.5 \mathrm{ppm})$. Coupling constants $(J)$ are expressed in Hertz $(\mathrm{Hz})$. High-resolution mass spectrometry (HRMS) was performed on a Finnigan MAT 8400 mass spectrometer using electron impact ionization (EI). Size exclusion chromatography (SEC) was performed using a Waters 515 HPLC pump, two PLgel mixed-D columns $(5 \mu \mathrm{m}$ pore size, $300 \mathrm{~mm} \times 7.5 \mathrm{~mm}$ ) and their corresponding guard column, and a Wyatt Optilab rEX refractive index detector. DMF with $1 \% \mathrm{NEt}_{3}$ and $10 \mathrm{mM}$ $\mathrm{LiBr}$ was used as the eluent. The column temperature was 85

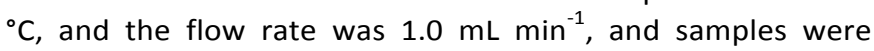


analyzed at a concentration of $5 \mathrm{mg} \mathrm{mL}^{-1}$. Molar mass was determined relative to PMMA standards. FT-IR spectra were recorded on either a Bruker Vector 33 instrument in transmission mode, or a Perkin Elmer Spectrum Two instrument with a diamond universal ATR attachment. UV-vis spectroscopy was performed using a Varian Cary 300 Bio UVvisible spectrophotometer.

\section{Synthesis of (E)-(4-((2-chlorophenyl)diazenyl)phenyl)methanol (Compound 2) and representative synthesis of an azobenzene}

2-Chloroaniline (1.00 g, $7.84 \mathrm{mmol}$ ) was dissolved in $\mathrm{CH}_{2} \mathrm{Cl}_{2}(20$ $\mathrm{mL})$ and $\mathrm{H}_{2} \mathrm{O}(50 \mathrm{~mL})$ was added to the solution. A solution of oxone $(9.64 \mathrm{~g}, 15.6 \mathrm{mmol})$ in water $(100 \mathrm{~mL})$ was added and the mixture was stirred for 2 hours at room temperature yielding a green solution. The solution was separated and the aqueous layer was extracted with $\mathrm{CH}_{2} \mathrm{Cl}_{2}(100 \mathrm{~mL})$. The organic phase was washed with $1 \mathrm{M} \mathrm{HCl}(100 \mathrm{~mL})$, saturated $\mathrm{NaHCO}_{3}$ $(100 \mathrm{~mL})$ and saturated $\mathrm{NaCl}(100 \mathrm{~mL})$, then dried on $\mathrm{MgSO}_{4}$ and filtered. This solution was used immediately without further purification. $\mathrm{AcOH}(5 \mathrm{~mL})$ was added to the $\mathrm{CH}_{2} \mathrm{Cl}_{2}$ solution. 4-Aminobenzyl alcohol (1.06 g, $8.62 \mathrm{mmol}$ ) was added and the mixture was stirred at room temperature for 16 hours. The organic phase was washed with saturated $\mathrm{NaHCO}_{3}$ $(100 \mathrm{~mL})$, and saturated $\mathrm{NaCl}(100 \mathrm{~mL})$. The organic phase was then dried on $\mathrm{MgSO}_{4}$, filtered and concentrated in vacuo. The collected solid was then purified by column chromatography (EtOAc/Hex 40:60) to provide compound 2, as an orange solid (1.78 g, 92\%). ${ }^{1} \mathrm{H}$ NMR $\left(400 \mathrm{MHz}, \mathrm{CDCl}_{3}\right): \delta=4.80(\mathrm{~s}, 2 \mathrm{H})$, 7.33$7.38(\mathrm{~m}, 1 \mathrm{H}), 7.39-7.43(\mathrm{~m}, 1 \mathrm{H}), 7.52-7.54(\mathrm{~m}, 2 \mathrm{H}), 7.56-7.58$ $(m, 1 \mathrm{H}), 7.70-7.72(m, 1 \mathrm{H}), 7.97-7.99(\mathrm{~m}, 2 \mathrm{H}) .{ }^{13} \mathrm{C}$ NMR $(100$ $\mathrm{MHz}): \delta=152.2,148.7,144.4,135.3,131.6,130.7,127.4$, 127.3, 123.6, 117.6, 64.8. FT-IR ( NaCl, thin film, $\left.v_{\max } / \mathrm{cm}^{-1}\right)$ : $3303(\mathrm{O}-\mathrm{H}), 1219(\mathrm{~N}=\mathrm{N}), 1030(\mathrm{C}-\mathrm{O}$, alcohol). HRMS (EI): calc. for $\mathrm{C}_{13} \mathrm{H}_{11} \mathrm{ClN} \mathrm{N}_{2} \mathrm{O}+[\mathrm{M}]+:$ 246.0560, found 246.0556.

\section{Synthesis of Polymer P1}

Compound 18 (160 mg, $0.323 \mathrm{mmol}$ ) and compound $19^{36}$ (16 $\mathrm{mg}, 0.036 \mathrm{mmol})$ were dissolved in distilled DMA $(600 \mu \mathrm{L})$. Compound $20^{37}$ (129 mg, $0.359 \mathrm{mmol}$ ) was then added to this solution. $\mathrm{NEt}_{3}(115 \mu \mathrm{L}, 0.825 \mathrm{mmol})$ was added and the resulting mixture was stirred for 48 hours. The resulting gel was then dissolved by dilution with DMA $(1 \mathrm{~mL})$ and dialysed in against DMF and then water using a membrane with a $1 \mathrm{~kg}$ $\mathrm{mol}^{-1} \mathrm{MWCO}$. Lyophilization provided $\mathbf{P 1}$ as an orange solid (135 mg, 65\%). ${ }^{1} \mathrm{H}$ NMR (600 MHz, DMSO- $\left.d_{6}\right): \delta=1.24-1.37(\mathrm{~m}$, $1.75 \mathrm{H}), 1.61$ (br s, 0.29H), 2.35-2.37 (m, 4H), 2.61-2.72 (m, $0.47 \mathrm{H}), 3.83-3.87(\mathrm{~m}, 3.57 \mathrm{H}), 4.00-4.05(\mathrm{br} \mathrm{s}, 0.35 \mathrm{H}), 4.82-4.84$ $(\mathrm{m}, 0.52 \mathrm{H}), 5.02-5.09(\mathrm{~m}, 2.43 \mathrm{H}), 5.19-5.21$ (br s, 2.64H), 6.61$6.63(\mathrm{~m}, 0.25 \mathrm{H}), 6.93-6.95(\mathrm{~m}, 0.53 \mathrm{H}), 7.18-7.24(\mathrm{~m}, 1.42 \mathrm{H})$, 7.36-7.57 (m, 3.89), 7.69-7.72 (m, 2.38H), 7.95-7.97 (br s, $1.18 \mathrm{H}), 8.32-8.35(\mathrm{~m}, 1.70 \mathrm{H})$. SEC: $\mathrm{M}_{\mathrm{W}}=7200 \mathrm{~g} \mathrm{~mol}^{-1}, \Theta=2.26$.

\section{Synthesis of Polymer P2}

P1 (50 mg) was dissolved in $\mathrm{CH}_{2} \mathrm{Cl}_{2}(5 \mathrm{~mL})$. TFA $(5 \mathrm{~mL})$ was then added and the reaction mixture was stirred for 1.5 hours. The solution was then concentrated in vacuo to give an orange solid. This was dissolved in DMA (1 mL) at room temperature. EDC. $\mathrm{HCl}(5.02 \mathrm{mg}, 26.2 \mu \mathrm{mol})$ was then added to this solution, followed by PEO-NH $\mathrm{N}_{2}\left(2000 \mathrm{~g} \mathrm{~mol}^{-1}, 52.4 \mathrm{mg}, 26.2 \mu \mathrm{mol}\right)$. Catalytic DIPEA $(9 \mu \mathrm{L}, \sim 50 \mu \mathrm{mol})$ and DMAP $(0.2 \mathrm{mg}, \sim 2 \mu \mathrm{mol})$ were added and the reaction mixture was stirred for 24 hours. The solution was then dialysed using a $50 \mathrm{~kg} \mathrm{~mol}^{-1} \mathrm{MWCO}$ dialysis tubing in DMF $(50 \mathrm{~mL})$ and then water $(50 \mathrm{~mL})$. The polymer solution was lyophilized to give $\mathbf{P 2}$ as an orange solid (60 mg, 70\%). ${ }^{1} \mathrm{H}$ NMR $\left(600 \mathrm{MHz}, \mathrm{DMSO}-d_{6}\right): \delta=2.35-2.36(\mathrm{~m}$, $4.40 \mathrm{H}), 2.61-2.72(\mathrm{~m}, 0.57 \mathrm{H}), 3.24(\mathrm{~s}, 1.02 \mathrm{H}), 3.4-3.6(\mathrm{~m}$, $50.47 \mathrm{H}), 3.82-3.88(\mathrm{~m}, 3.60 \mathrm{H}), 3.95-4.05(\mathrm{~m}, 0.35 \mathrm{H}), 4.81-4.83$ (br s, 0.49H), 5.01-5.09 (m, 2.46H), 5.19-5.21 (br s, 2.66H), 6.62-6.64 (m, 0.34H), 6.93-6.96 (br s, 0.51H), 7.17-7.24 (m, $1.49 \mathrm{H}), 7.36-7.58(\mathrm{~m}, 4.34 \mathrm{H}), 7.69-7.72(\mathrm{br} \mathrm{s}, 2.73 \mathrm{H}), 7.95-7.97$ (br s, 1.26H), 8.13-8.15 (br s, 0.17H), 8.32-8.34 (br s, 1.80H). SEC: $\mathrm{M}_{\mathrm{W}}=5900 \mathrm{~g} \mathrm{~mol}^{-1}, \oplus=2.13$.

\section{Determination of $\mathbf{k}_{\mathrm{obs}}$ for the Reduction of Azobenzenes 1-12.}

Solutions of azobenzenes $\mathbf{1}-\mathbf{1 2}$ at $25 \mu \mathrm{M}$ in THF/water (3/5) were prepared. To a solution of $5.0 \mathrm{~mL}$ of each azobenzene was added $100 \mu \mathrm{L}$ of hydrazine hydrate $\left(\sim 50 \% \mathrm{~N}_{2} \mathrm{H}_{4}\right)$ to achieve a concentration of $\sim 250 \mu \mathrm{M}$. Absorption spectra were obtained initially and then every minute for 10 minutes over a wavelength range of $200-500 \mathrm{~nm}$ in a quartz cuvette with a 1 $\mathrm{cm}$ path length. $\mathrm{k}_{\mathrm{obs}}$ was determined as the negative slope of the plot of $\ln \left(A / A_{0}\right)$ versus time. The experiment was repeated in triplicate for each azobenzene and the error reported on $k_{\text {obs }}$ corresponds to the standard deviation of the three values.

\section{Formation of Aqueous Polymer Assemblies}

A solution of $\mathbf{P 2}$ or $\mathbf{P 3}$ was prepared in DMSO at a concentration of $8.0 \mathrm{mg} \mathrm{mL}$.-1 $0.1 \mathrm{~mL}$ of this solution was added rapidly to $0.9 \mathrm{~mL}$ of stirring deionized water. The resulting solution was stirred for approximately 30 minutes, and then diluted four-fold by the addition of $3.0 \mathrm{~mL}$ of $\mathrm{DI}$ water. The resulting solution was then dialyzed in $1 \mathrm{~L}$ of DI water to remove the organic solvent, using a $50 \mathrm{~kg} \mathrm{~mol}^{-1}$ MWCO membrane for at least 16 hours, to yield $4.0 \mathrm{~mL}$ of polymer assemblies at a concentration of $0.2 \mathrm{mg} \mathrm{mL}^{-1}$.

\section{Encapsulation of Nile Red}

A stock solution of nile red in THF $(0.16 \mathrm{mg} / \mathrm{mL})$ was prepared. To a clean dry vial was added $0.1 \mathrm{~mL}$ of the nile red solution, and the THF was evaporated under a stream of air. The resulting nile red residue was then re-dissolved in $0.1 \mathrm{~mL}$ of 8.0 $\mathrm{mg} \mathrm{mL}^{-1}$ polymer solution in DMSO, and this solution was used in the formation of polymer assemblies as described above. This provided polymer assemblies with 5 wt\% encapsulated nile red dye relative to the polymer mass, at a polymer concentration of $0.2 \mathrm{mg} \mathrm{mL}^{-1}$.

\section{Transmission Electron Microscopy (TEM)}

Transmission electron microscopy was performed using a Phillips CM10 microscope operating at $80 \mathrm{kV}$ with a $40 \mu \mathrm{m}$ aperture. Samples were prepared at a concentration of 0.05 
$\mathrm{mg} \mathrm{mL}^{-1}$ and $10 \mu \mathrm{L}$ of solution was placed on a 400-mesh copper grid with a formvar coating from Electron Microscopy Sciences and allowed to dry overnight prior to imaging.

\section{Dynamic Light Scattering Studies}

Dynamic light scattering was performed using a Malvern Zetasizer Nano ZS equipped with a $633 \mathrm{~nm}$ laser, using a scattering angle of $173^{\circ}$. For all studies aimed at determining the time-dependent effects of stimuli on the mean count rate of the samples, the attenuator value was fixed according to the computer-optimized attenuator value for the initial assemblies. The temperature was set to $25^{\circ} \mathrm{C}$, and the samples were equilibrated at this temperature for at least 30 seconds prior to measurements. Each measurement was the average of more than 10 scans of the same sample, and three separate samples were measured at each time point.

\section{Fluorescence Studies}

Fluorescence emission spectroscopy was carried out using a Photon Technology International QM-4 SE spectrofluorometer. The excitation wavelength was $485 \mathrm{~nm}$ and the emission spectrum was measured between 520 and $700 \mathrm{~nm}$. The fluorescence was measured at the maximum emission wavelength.

\section{Procedure for Alternating Irradiation with UV and Visible Light}

The UV light source consisted of 16 UVA bulbs, (Hitachi FL8BL$B, 8$ watts) with emission centred at $365 \mathrm{~nm}$, set at a distance of $10 \mathrm{~cm}$ from the sample. The visible light source was a 1 watt white LED bulb set at a distance of $1 \mathrm{~cm}$ from the sample. 1.0 $\mathrm{mL}$ of the $0.2 \mathrm{mg} \mathrm{mL}^{-1}$ assemblies prepared with or without encapsulated nile red as described above was alternately irradiated in a quartz cuvette with UV light for 10 minutes, followed by visible light for 10 minutes. The process was repeated four times. The sample without nile red was analysed by UV-Vis spectroscopy after each irradiation whereas the sample containing nile red was analysed by fluorescence spectroscopy after each irradiation.

\section{Procedure for Constant Irradiation with UV light}

$1.0 \mathrm{~mL}$ of the $0.2 \mathrm{mg} / \mathrm{mL} \mathbf{P 2}$ nanoassembly sample with (for fluorescence studies) or without (for DLS studies) encapsulated nile red was placed in a quartz cuvette and irradiated continuously over 9 hours with the UV light source described above. The temperature was maintained at $\sim 22{ }^{\circ} \mathrm{C}$. At various time points, the sample was briefly removed from the light source and the DLS or fluorescence measurement was performed as described above. The control consisted of $\mathbf{P 2}$ micelles that were not irradiated with light. The experiments were performed in triplicate and error bars represent the standard deviations on three measurements.

\section{Procedure for Hydrazine-Induced Degradation of Assemblies}

To $1.0 \mathrm{~mL}$ of the $0.2 \mathrm{mg} \mathrm{mL}^{-1} \mathbf{P 2}$ or $\mathbf{P 3}$ nanoassembly sample with (for fluorescence studies) or without (for DLS studies) encapsulated nile red was added $20 \mu \mathrm{L}$ of hydrazine hydrate $\left(\sim 50 \% \mathrm{~N}_{2} \mathrm{H}_{4}\right)$. The sample was stirred at room temperature $\left(\sim 22{ }^{\circ} \mathrm{C}\right)$ and then analyzed by fluorescence measurement or DLS as described above at various time points. The experiments were performed in triplicate and error bars represent the standard deviations on three measurements.

\section{Procedure for Hydrazine-Induced Degradation of Assemblies, with UV Light Irradiation}

To $1.0 \mathrm{~mL}$ of the $0.2 \mathrm{mg} \mathrm{mL}^{-1} \mathbf{P} \mathbf{2}$ or $\mathbf{P} \mathbf{3}$ nanoassembly sample with (for fluorescence studies) or without (for DLS studies) encapsulated nile red in a quartz cuvette was added $20 \mu \mathrm{L}$ of hydrazine hydrate. The sample was then irradiated with the UV light source described above. The temperature was maintained at $\sim 22{ }^{\circ} \mathrm{C}$. At various time points, the sample was briefly removed from the light source and the DLS or fluorescence measurement was performed as described above. The experiments were performed in triplicate and error bars represent the standard deviations on three measurements.

\section{Acknowledgements}

The authors thank the Natural Sciences and Engineering Research Council of Canada for funding this work (Discovery Grant to ERG, CGSD award to ADW).

\section{Notes and references}

1 M. Cohen Stuart, W. T. S. Huck, J. Genzer, M. Muller, C. Ober, M. Stamm, G. B. Sukhorukov, I. Szieifer, V. V. Trukruk, M. Urban, F. Winnik, S. Zauscher, I. Luzinov and S. Minko, Nat. Mater., 2010, 9, 101-113.

2 X. Yan, F. Wang, B. Zheng and F. Huang, Chem. Soc. Rev., 2012, 41, 6042-6065.

3 Q. Li, ed., Intelligent Stimuli-Responsive Materials: From Well-Defines Nanostructures to Applications, John Wiley \& Sons, Inc., Hoboken, NJ, 2013.

4 J.-F. Gohy and Y. Zhao, Chem. Soc. Rev., 2013, 42, 71177129.

5 H. Zhao, E. S. Sterner, E. B. Coughlin and P. Theato, Macromolecules, 2012, 45, 1723-1736.

6 M. Huo, J. Yuan, L. Tao and Y. Wei, Polym. Chem., 2014, 5, 1519-1528.

7 F. Meng, W. E. Hennick and Z. Zhong, Biomaterials, 2009, 30, 2180-2198.

8 W. Gao, J. M. Chan and O. C. Farokhzad, Mol. Pharm., 2010, 7, 1913-1920.

9 Y. Bae and K. Kataoka, Adv. Drug Delivery Rev., 2009, 61, 768-784.

10 X. Guo, C. Shi, J. Wang, S. Di and S. Zhou, Biomaterials, 2013, 34, 4544-4554.

11 S. Strandman and X. X. Zhu, Prog. Polym. Sci., 2015, 42, 154-176. 
S. R. Abulateefeh, S. G. Spain, J. W. Aylott, W. C. Chan, M. C. Garnett and C. Alexander, Macromol. Biosci., 2011, 11, 1722-1734.

J. Thévenot, $H$. Oliveira, O. Sandre and $S$. Lecommandoux, Chem. Soc. Rev., 2013, 42, 7099-7116. S. Mura, J. Nicolas and P. Couvreur, Nat. Mater., 2013, 12, 991-1003.

Q. Yin, J. Shen, Z. Zhang, H. Yu and Y. Li, Adv. Drug 41 Delivery Rev., 2013, 65, 1699-1715.

P. Schattling, F. D. J. and P. Theato, Polym. Chem., 2014, 5, 25-36.

J. Zhuang, M. R. Gordon, J. Ventura, L. Li and S. 43 Thayumanavan, Chem. Soc. Rev., 2013, 42, 7421-7435.

A. A. Beharry and G. A. Woolley, Chem. Soc. Rev., 2011, 40, 4422-4437.

M. Dong, A. Babalhavaeji, S. Samanta, A. A. Beharry and G. A. Woolley, Acc. Chem. Res., 2015, 48, 26622670.

S. Yagai, T. Karatsu and A. Kitamura, Chemistry, 2005, 11, 4054-4063.

E. Merino and M. Ribagorda, Beilstein J. Org. Chem., 2012, 8, 1071-1090.

Y. Zhao and J. He, Soft Matter, 2009, 5, 2686-2693.

G. Wang, X. Tong and Y. Zhao, Macromolecules, 2004, 37, 8911-8917.

X. Tong, G. Wang, A. Soldera and Y. Zhao, J. Phys. Chem. B, 2005, 109, 20281-20287.

R. Dong, B. Zhu, Y. Zhou, D. Yan and X. Zhu, Polym. Chem., 2013, 4, 912.

J. del Barrio, L. Oriol, C. Sanches, J. L. Serrano, A. Di Cicco, P. Keller and M.-H. Li, J. Am. Chem. Soc., 2010, 132, 3762-3769.

A. D. Wong, T. M. Güngör and E. R. Gillies, ACS Macro Lett., 2014, 3, 1191-1195.

A. C. Fonseca, M. H. Gil and P. N. Simões, Prog. Polym. Sci., 2014, 39, 1291-1311.

A. Rodriguez-Galan, L. Franco and J. Puiggali, Polymer, 54 2011, 3, 65-99.

A. Soleimani, S. Drappel, R. Carlini, A. Goredema and E. 55

R. Gillies, Ind. Eng. Chem. Res., 2014, 53, 1452-1460.

Y. Saotome, T. Miyazawa and T. Endo, Chem. Lett., 56 1991, 1, 21-24.

J. Montane, E. Armelin, A. Rodriguez-Galan and J. Puiggali, J. Appl. Polym. Sci., 2002, 85, 1815-1824.

E. Armelin, N. Paracuellos, A. Rodriguez-Galan and J. Puiggali, Polymer, 2001, 42, 7923-7932.

N. Parades, A. Rodriguez-Galan and J. Puiggali, J. Polym. Sci., Part A: Polym. Chem., 1998, 36, 1271-1282.

R. Katsarava, V. Beridze, N. Arabull, D. Kharadze, C. C. Chu and C. Y. Won, J. Polym. Sci. Part A: Polym. Chem., 1999, 37, 391-407.

K. M. Atkins, D. Lopez, D. K. Knight, K. Mequanint and E. R. Gillies, J. Polym. Sci. A: Polym. Chem., 2009, 47, 3757-3772.

K. Guo and C. C. Chu, J. Polym. Sci. Part A: Polym Chem 2007, 45, 1595-1606.
K. Guo, C. C. Chu, E. Chkhaidze and R. Katsarava, J. Poly. Sci. A: Polym. Chem., 2005, 43, 1463-1477.

M. A. Dewit, Z. Wang, K. M. Atkins, K. Mequanint and E. R. Gillies, J. Polym. Sci. Part A: Polym. Chem., 2008, 46, 6376-6392.

J. Wu and C. C. Chu, J. Mater. Chem. B, 2013, 1, 353360.

D. K. Knight, E. R. Gillies and K. Mequanint, Biomacromolecules, 2011, 12, 2475-2487.

D. K. Knight, E. R. Gillies and K. Mequanint, Acta Biomater., 2014, 10, 3484-3496.

D. K. Knight, R. Stutchbury, D. Imruck, C. Halfpap, S. Lin, U. Langbein, E. R. Gillies, S. Mittler and K. Mequanint, ACS Appl. Mater. Interfaces, 2012, 4, 1303-1312.

M. Deng, J. Wu, C. A. Reinhart-King and C. C. Chu, Biomacromolecules, 2009, 10, 3037-3047.

K. Guo and C. C. Chu, J. Biomed. Mater. Res., Part B., 2008, 89B(2), 491-500.

J. A. Horwitz, K. M. Shum, J. C. Bodle, M. Deng and C. C. Chu, J. Biomed. Mat. Res. A, 2010, 95A, 371-380.

S. H. Lee, I. Szinai, K. Carpenter, R. Katsarava, G. Jokhadze, C. C. Chu, Y. Huang, E. Verbeken, O. Bramwel, I. De Scheerder and M. K. Hong, Coronary Artery Dis., 2002, 13, 237-241.

D. Yamanouchi, J. Wu, A. N. Lazar, K. C. Kent, C. C. Chu and B. Liu, Biomaterials, 2008, 29, 3269-3277.

J. Wu, D. Yamanouchi, B. Liu and C. C. Chu, J. Mater. Chem., 2012, 22, 18983-18991.

G. J. Zilinskas, A. Soleimani and E. R. Gillies, Int. J. Poly. Sci., 2012, 2012, 1-11.

A. Soleimani, A. Borecki and E. R. Gillies, Polym. Chem., 2014, 5, 7062-7071.

A. Soleimani, M. M. A. R. Moustafa, A. Borecki and E. R. Gillies, Can. J. Chem., 2014, 93, 399-405.

M. A. DeWit and E. R. Gillies, J. Am. Chem. Soc., 2009, 131, 18327-18334.

R. A. McBride and E. R. Gillies, Macromolecules, 2013, 46, 5157-5166.

W. M. Koppes, J. S. Moran, J. C. Oxley and J. L. Smith, Tet. Lett., 2008, 49, 3234-3237.

T. E. Brown, H. E. LeMay, B. E. Bursten, C. Murphy, P. Woodward and M. E. Stoltzfus, Chemistry: The Central Science, Prentice Hall, 13 edn., 2014.

C. Hansch, A. Leo and R. W. Taft, Chem. Rev., 1991, 91, 165-195.

R. Minkwitz and M. Berkei, Inorg. Chem., 1999, 38, 5041-5044.

R. Wilcken, M. O. Zimmermann, A. Lange, A. C. Joerger and F. M. Boeckler, J. Med. Chem., 2013, 56, 13631388.

P.-H. Lin, M. Leclere, J. Long, T. J. Burchell, I. Korobkov, R. Clerac and M. Murugesu, Dalton Trans., 2010, 39, 5698-5704.

X. Jiang, E. B. Vogel, M. R. Smith, III and G. L. Baker, Macromolecules, 2008, 41, 1937-1944.

D. K. Knight, E. R. Gillies and K. Mequanint, Acta Biomater, 2014, 10, 3484-3496. 
63 P. Greenspan, E. P. Mayer and S. D. Fowler, J. Cell Biol., 1985, 100, 965-973.

64 M. M. G. Krishna, J. Phys. Chem. A, 1999, 103, 35893595.

65 J. Rao, C. Hottinger and A. Khan, J. Am. Chem. Soc., 2014, 136, 5872-5875. 